TAIWANESE JOURNAL OF MATHEMATICS

Vol. 16, No. 3, pp. 941-962, June 2012

This paper is available online at http://journal.taiwanmathsoc.org.tw

\title{
EXISTENCE AND STABILITY OF SOLUTIONS FOR GENERALIZED SYMMETRIC STRONG VECTOR QUASI-EQUILIBRIUM PROBLEMS
}

\author{
Bin Chen, Nan-jing Huang and Ching-Feng Wen*
}

\begin{abstract}
In this paper, a class of generalized symmetric strong vector quasiequilibrium problems in real locally convex Hausdorff topological vector spaces is studied. By using the Kakutani-Fan-Glicksberg fixed point theorem, an existence theorem of solutions for the generalized symmetric strong vector quasi-equilibrium problems is obtained. Moreover, the closedness of the solution set and a stability result of solutions for such problem are also derived.
\end{abstract}

\section{INTRODUCTION}

The equilibrium problem contains many important problems as special cases, such as optimization problems, problems of Nash equilibrium, fixed point problems, variational inequalities and complementarity problems. In recent years, there has been an increasing interest in the study of vector equilibrium problems. A lot of existence results of solutions for vector equilibrium problems and vector variational inequalities have been established (see, e.g., $[2,4,10,17]$ and the references therein).

In 1994, Noor and Oettli [34] introduced and studied the symmetric quasi-equilibrium problem which is a generalization of equilibrium problem proposed by Blum and Oettli [7]. In 2003, Fu[19] introduced the symmetric vector quasi-equilibrium problem which is a generalization of the symmetric quasi-equilibrium problem proposed by Noor and Oettli [34] and gave an existence theorem for weak Pareto solution for the symmetric vector quasi-equilibrium problem in Hausdorff locally convex spaces. Farajzadeh [16] supplied a further extension to Hausdorff topological vector spaces with several assumptions being relaxed. Anh and Khanh [2] extended the problem considered in Noor and Oettli [34], Fu [19] and Farajzadeh [16] from the single-valued case to the

Received December 10, 2010, accepted March 29, 2011.

Communicated by Jen-Chih Yao.

2010 Mathematics Subject Classification: 49J40, 90C29.

Key words and phrases: Generalized symmetric strong vector quasi-equilibrium problems, Stability, Kakutani-Fan-Glicksberg fixed point theorem.

This work was supported by the Key Program of NSFC (Grant No. 70831005), the National Natural Science Foundation of China (10671135) and NSC 99-2115-M-037-001.

*Corresponding author. 
multivalued case in Hausdorff topological vector spaces. Gong [23] introduced the symmetric strong vector quasi-equilibrium problem and gave an existence theorem of strong efficient solution for symmetric vector quasi-equilibrium problem in Hausdorff locally convex spaces. It is well known that a strong efficient solution of a vector equilibrium problem is an idea solution. It is better than other solutions such as efficient solution, weak efficient solution, proper efficient solution and supper efficient solution(see [24]). Hence, it is important to study the existence of strong efficient solution and properties of the strong efficient solution set. Recently, Hou, Gong and Yang [26] derived an existence theorem of strong efficient solution for generalized strong vector quasi-equilibrium problem and discussed the stability of strong efficient solutions. Long, Huang and Teo [33], Yu and Gong [37] extended the main results of Hou, Gong and Yang [26] from single-valued mappings to set-valued mappings.

On the other hand, one of important problems of vector equilibrium is to investigate the stability of solutions. For the stability of symmetric equilibrium problems, there have been limited number of works in the literature. Recently, Anh and Khanh [3] derived various kinds of semicontinuity for the solution sets of parametric multivalued symmetric vector quasi-equilibrium problems. Chen and Gong [12] studied the stability of the solutions set for symmetric vector quasi-equilibrium problems. To the best of our knowledge, no paper has been devoted to the study of stability for generalized symmetric strong vector quasi-equilibrium problems.

Motivated and inspired by the research works mentioned above, in this paper, we consider a class of generalized symmetric strong vector quasi-equilibrium problems in real locally convex Hausdorff topological vector spaces. We establish an existence theorem of solutions by using Kakutani-Fan-Glicksberg fixed point theorem and discuss the closedness of the solution set for the generalized symmetric strong vector quasiequilibrium problems. Moreover, we also show a stability result for such problem.

\section{Preliminaries Results}

Throughout this paper, unless specified otherwise, we suppose that $X, Y$ and $Z$ be real locally convex Hausdorff topological vector spaces and $C \subset Z$ be a closed convex cone. The convex cone induces a partially ordering in $Z$, defined by

$$
z_{1} \leq z_{2}\left(\text { or } z_{2} \geq z_{1}\right) \text { if and only if } z_{2}-z_{1} \in C \text {. }
$$

Let $E$ be a a nonempty subset of $X$, and $D$ be a nonempty subset of $Y$. Let $S$ : $E \times D \rightarrow 2^{E}, T: E \times D \rightarrow 2^{D}, F: E \times D \times E \rightarrow 2^{Z}$ and $G: D \times E \times D \rightarrow 2^{Z}$ be four set-valued mappings.

In this paper, we consider the following generalized symmetric strong vector quasiequilibrium problem(in short, GSSVQEP): finding $(\bar{x}, \bar{y}) \in E \times D$ such that $\bar{x} \in$ $S(\bar{x}, \bar{y}), \bar{y} \in T(\bar{x}, \bar{y})$ and 


$$
\begin{aligned}
& F(\bar{x}, \bar{y}, x) \subset C, \forall x \in S(\bar{x}, \bar{y}), \\
& G(\bar{y}, \bar{x}, y) \subset C, \forall y \in T(\bar{x}, \bar{y}) .
\end{aligned}
$$

For our main results, we need some definitions and lemmas as follows.

Definition 2.1. ([1]). Let $X$ and $Y$ be two topological vector spaces, and $T: X \rightarrow$ $2^{Y}$ be a set-valued mapping.

(i) $T$ is said to be upper semicontinuous at $x \in X$ if, for any neighborhood $U$ of $T(x)$, there is a neighborhood $V$ of $x$ such that

$$
T(t) \subset U, \forall t \in V .
$$

$T$ is said to be upper semicontinuous on $X$ if it is upper semicontinuous at each $x \in X$

(ii) $T$ is said to be lower semicontinuous at $x \in X$ if, for any $y \in T(x)$ and for any net $\left\{x_{\alpha}\right\}$ converging to $x$, there exists a net $\left\{y_{\alpha}\right\}$ such that $y_{\alpha} \in T\left(x_{\alpha}\right)$ and $\left\{y_{\alpha}\right\}$ converges to $y$.

$T$ is said to be lower semicontinuous on $X$ if it is lower semicontinuous at each $x \in X$.

(iii) $T$ is said to be continuous on $X$ if it is both upper semicontinuous and lower semicontinuous on $X$.

(iv) $T$ is said to be closed, if $\operatorname{Graph}(T)=\{(x, y): x \in X, y \in T(x)\}$ is a closed subset in $X \times Y$.

Definition 2.2. Let $W$ be a topological vector space and $D \subset W$ be a nonempty set. A set-valued mapping $G: D \rightarrow 2^{Z}$ is said to be $C$-upper semicontinuous on $x_{0}$ if, for any neighborhood $U$ of 0 in $Z$, there exists a neighborhood $U\left(x_{0}\right)$ of $x_{0}$ such that

$$
G(x) \subset G\left(x_{0}\right)+U+C, \forall x \in U\left(x_{0}\right) \cap D .
$$

Definition 2.3. Let $W$ be a topological vector space and $D \subset W$ be a nonempty set. A set-valued mapping $G: D \rightarrow 2^{Z}$ is said to be $C$-lower semicontinuous on $x_{0}$ if, for each $z \in G\left(x_{0}\right)$, and any neighborhood $U$ of 0 in $Z$, there exists a neighborhood $U\left(x_{0}\right)$ of $x_{0}$ such that

$$
G(x) \cap(z+U-C) \neq \emptyset, \forall x \in U\left(x_{0}\right) \cap D .
$$

Definition 2.4. Let $W$ be a topological vector space and $D \subset W$ be a nonempty set. A set-valued mapping $G: D \rightarrow 2^{Z}$ is said to be $C$-quasiconvex if, for any $z \in W$, $x_{1}, x_{2} \in D, t \in[0,1], z_{1} \in G\left(x_{1}\right), z_{2} \in G\left(x_{2}\right)$ and $z_{1} \leq z, z_{2} \leq z$, there exist $z_{t} \in G\left(t x_{1}+(1-t) x_{2}\right)$ such that $z_{t} \leq z$. 
Definition 2.5. Let $W$ be a topological vector space and $D \subset W$ be a nonempty set. A set-valued mapping $G: D \rightarrow 2^{Z}$ is said to be $C$-properly quasiconvex if, for any $x, y \in D, t \in[0,1], u \in G(x), v \in G(y)$, there exists $z \in G(t x+(1-t) y)$ such that either $z \leq u$ or $z \leq v$.

Lemma 2.1. ([1]). Let $X$ and $Y$ be two Hausdorff topological vector spaces and $T: X \rightarrow 2^{Y}$ be a set-valued mapping.

(i) If $T$ is upper semicontinuous with closed values, then $T$ is closed.

(ii) If $T$ is closed and $Y$ is compact, then $T$ is upper semicontinuous.

Lemma 2.2. (Kakutani-Fan-Glicksberg [25]). Let $X$ be a locally convex Hausdorff topological vector space and $K$ be a nonempty compact convex subset of $X$. Let $T: K \rightarrow 2^{K}$ be a upper semicontinuous set-valued mapping with nonempty closed convex values. Then there exists $\bar{x} \in K$ such that $\bar{x} \in T(\bar{x})$.

Lemma 2.3. Let $D$ be a nonempty convex subset of $Z$ and $G: D \rightarrow 2^{Z}$ be $C$-upper semicontinuous mapping with compact values such that for every $x \in D$, there exists $z_{0} \in G(x)$ such that $z_{0} \leq z$ for all $z \in G(x)$. Then $G$ is $C$-properly quasiconvex if and only if the following conditions hold:

(i) For any $x, y \in D$, there exists $t_{0} \in[0,1]$ such that for all $u \in G(x)$ and $v \in G(y)$, there exists $z \in G\left(t_{0} x+\left(1-t_{0}\right) y\right)$ with $z \leq u$ and $z \leq v$;

(ii) $G$ is $C$-quasiconvex.

Proof. The necessity see the Lemma 2.1 of [37]. Next we prove the sufficiency, i.e. if the conditions (i) and (ii) are satisfied, then $G$ is $C$-properly quasiconvex.

If $t_{0}=1$, then by (i), for $u \in G(x), v \in G(y)$, there exists $z \in G(x)$ such that $z \leq u$ and $z \leq v$. By the assumption, there exists $z_{1} \in G(y)$ such that $z_{1} \leq v$. By (ii), there exists $z_{t} \in G(t x+(1-t) y)$ such that $z_{t} \leq v$.

If $t_{0}=0$, then it follows from (i) that, for $u \in G(x), v \in G(y)$, there exists $z \in G(y)$ such that $z \leq u$ and $z \leq v$. By the assumption, there exists $z_{2} \in G(x)$ such that $z_{2} \leq u$. The condition (ii) implies that there exists $z_{t} \in G(t x+(1-t) y)$ such that $z_{t} \leq u$.

If $t_{0} \in(0,1)$, then for $u \in G(x), v \in G(y)$ and $t \in[0,1]$, when $t \geq t_{0}$, take $\alpha=\left(t-t_{0}\right) /\left(1-t_{0}\right) \in[0,1]$. Letting $x_{t_{0}}=t_{0} x+\left(1-t_{0}\right) y$, the condition (i) implies that there exists $z \in G\left(x_{t_{0}}\right)$ such that $z \leq u$ and $z \leq v$. By the assumption, there exists $z_{1} \in G(x)$ such that $z_{1} \leq u$. It follows from (ii) that there exists $z_{t} \in$ $G\left(\alpha x+(1-\alpha) x_{t_{0}}\right)=G(t x+(1-t) y)$ such that $z_{t} \leq u$. When $t<t_{0}$, take $\alpha=$ $\left(t_{0}-t\right) / t_{0} \in[0,1]$. Letting $x_{t_{0}}=t_{0} x+\left(1-t_{0}\right) y$, by (i), there exists $z \in G\left(x_{t_{0}}\right)$ such that $z \leq u$ and $z \leq v$. By the assumption, there exists $z_{2} \in G(y)$ such that $z_{2} \leq v$. The condition (ii) implies that there exists $z_{t} \in G\left(\alpha y+(1-\alpha) x_{t_{0}}\right)=G(t x+(1-t) y)$ such that $z_{t} \leq v$. 
Hence, for $u \in G(x), v \in G(y), t \in[0,1]$, there exists $z_{t} \in G(t x+(1-t) y)$ such that either $z_{t} \leq u$ or $z_{t} \leq v$. Thus, $G$ is $C$-properly quasiconvex.

\section{Existence OF SOlutions}

In this section, we establish an existence theorem of solutions by using KakutaniFan-Glicksberg fixed point theorem and discuss the closedness of the solution set for the generalized symmetric strong vector quasi-equilibrium problems.

Theorem 3.1. Let $X, Y, Z$ be real locally convex Hausdorff topological vector spaces, $E \subset X$ and $D \subset Y$ be nonempty compact convex sets. Let $S: E \times D \rightarrow 2^{E}$ and $T: E \times D \rightarrow 2^{D}$ be continuous set-valued mappings with nonempty compact convex values. Let $F: E \times D \times E \rightarrow 2^{Z}$ and $G: D \times E \times D \rightarrow 2^{Z}$ be $C$-upper semicontinuous and $C$-lower semicontinuous mappings with nonempty compact values. Assume that

(i) For each $\left(x, y, x^{\prime}\right) \in E \times D \times E$, there exists $z \in F\left(x, y, x^{\prime}\right)$ such that

$$
z \leq z^{\prime}, \forall z^{\prime} \in F\left(x, y, x^{\prime}\right)
$$

For each $\left(y, x, y^{\prime}\right) \in D \times E \times D$, there exists $z \in G\left(y, x, y^{\prime}\right)$ such that

$$
z \leq z^{\prime}, \forall z^{\prime} \in G\left(y, x, y^{\prime}\right)
$$

(ii) For all $(x, y) \in E \times D, F(x, y, x) \subset C$ and $G(y, x, y) \subset C$;

(iii) For all $(x, y) \in E \times D, F(x, y, u)$ is $C$-properly quasiconvex in $u$ and $G(y, x, v)$ is $C$-properly quasiconvex in $v$.

Then GSSVQEP has a solution. Moreover, the solution set of GSSVQEP is closed.

Proof. For any $(x, y) \in E \times D$, define $A: E \times D \rightarrow 2^{E}$ and $B: E \times D \rightarrow 2^{D}$ by

$$
\begin{aligned}
A(x, y)= & \{v \in S(x, y): \text { for any } u \in S(x, y), z \in F(x, y, u), \\
& \text { there exists } w \in F(x, y, v), \text { such that } w \leq z\}, \\
B(x, y)= & \{a \in T(x, y): \text { for any } b \in T(x, y), e \in G(y, x, b), \\
& \text { there exists } d \in G(y, x, a), \text { such that } d \leq e\} .
\end{aligned}
$$

(I) For any $(x, y) \in E \times D, A(x, y)$ is nonempty.

Indeed, for every $u \in S(x, y)$, set

$$
\begin{aligned}
H(u)= & \{v \in S(x, y): \text { for any } z \in F(x, y, u), \\
& \text { there exists } w \in F(x, y, v), \text { such that } w \leq z\} .
\end{aligned}
$$


Then $u \in H(u)$ and so $H(u) \neq \emptyset$.

Now we show the family $\{H(u): u \in S(x, y)\}$ has the finite intersection property. Letting $u_{1}, u_{2} \in S(x, y)$, by the assumptions and Lemma 2.3, there exists $t \in[0,1]$ such that for any $z_{1} \in F\left(x, y, u_{1}\right)$ and $z_{2} \in F\left(x, y, u_{2}\right)$, there exists $z \in F\left(x, y, t u_{1}+\right.$ $\left.(1-t) u_{2}\right)$ with

$$
z \leq z_{1} \text { and } z \leq z_{2} \text {. }
$$

From the convexity of $S(x, y)$, we know that $v:=t u_{1}+(1-t) u_{2} \in S(x, y)$ and so $v \in H\left(u_{1}\right) \cap H\left(u_{2}\right)$. Let $u_{1}, u_{2}, \cdots, u_{n} \in S(x, y)$ and $\bigcap_{i=1}^{n} H\left(u_{i}\right) \neq \emptyset$. Then, there exists $v \in \bigcap_{i=1}^{n} H\left(u_{i}\right)$. By the definition, $v \in S(x, y)$. For any $z_{i} \in F\left(x, y, u_{i}\right), i=$ $1,2, \cdots, n$, there exists $w_{i} \in F(x, y, v)$ such that

$$
w_{i} \leq z_{i} .
$$

By the assumptions, there exists $w \in F(x, y, v)$ such that $w \leq w_{i}$ for $i=1,2, \cdots, n$, It follows from (3.1) that

$$
w \leq z_{i}, i=1,2, \cdots, n
$$

Let $u_{n+1} \in S(x, y)$. For any $z_{n+1} \in F\left(x, y, u_{n+1}\right)$, by the assumptions and lemma 2.3, there exists $t \in[0,1]$ and $z_{0} \in F\left(x, y, t v+(1-t) u_{n+1}\right)$ such that

$$
z_{0} \leq w \text { and } z_{0} \leq z_{n+1} .
$$

From (3.2) and (3.3), we have

$$
z_{0} \leq z_{i}, i=1.2, \cdots, n+1 \text {. }
$$

By the convexity of $S(x, y)$, we have $t v+(1-t) u_{n+1} \in S(x, y)$ and so

$$
t v+(1-t) u_{n+1} \in \bigcap_{i=1}^{n+1} H\left(u_{i}\right) .
$$

Next, we show that $H(u)$ is closed. Let $\left\{v_{\alpha}: \alpha \in I\right\} \subset H(u)$ be a net such that $v_{\alpha} \rightarrow$ $v$. Then $\left\{v_{\alpha}\right\} \subset S(x, y)$ and for any $z \in F(x, y, u)$, there exists $w_{\alpha} \in F\left(x, y, v_{\alpha}\right)$ such that

$$
w_{\alpha} \leq z .
$$

It follows from the closedness of $S(x, y)$ that we have $v \in S(x, y)$. We claim that, for any $z \in F(x, y, u)$, there exists $w \in F(x, y, v)$ such that $w \leq z$. If not, there exists $z_{0} \in F(x, y, u)$ such that for any $w \in F(x, y, v)$,

$$
z_{0}-w \notin C \text {. }
$$


Hence,

$$
\left(z_{0}-F(x, y, v)\right) \cap C=\emptyset .
$$

Since $C$ is closed convex cone and $F(x, y, v)$ is compact, there exists some neighborhood $U$ of 0 such that

$$
\left(z_{0}-(F(x, y, v)+U+C)\right) \cap C=\emptyset .
$$

Since $v_{\alpha} \rightarrow v$ and $F(x, y, v)$ is $C$-upper semicontinuous in $v$, there exists $\alpha_{0} \in I$ such that for all $\alpha \geq \alpha_{0}$,

$$
F\left(x, y, v_{\alpha}\right) \subset F(x, y, v)+U+C .
$$

It follows from (3.5) that,

$$
\left(z_{0}-F\left(x, y, v_{\alpha}\right)\right) \cap C=\emptyset, \forall \alpha \geq \alpha_{0} .
$$

Because $z_{0} \in F(x, y, u)$, by (3.4), there exists $w_{\alpha} \in F\left(x, y, v_{\alpha}\right)$ such that $z_{0}-w_{\alpha} \in C$. This contradicts (3.6) and so $H(u)$ is closed.

Since $S(x, y)$ is closed and $E$ is compact, we know that $S(x, y)$ is compact. Hence,

$$
\bigcap_{u \in S(x, y)} H(u) \neq \emptyset
$$

Letting $v \in \bigcap_{u \in S(x, y)} H(u)$, then $v \in S(x, y)$, and for any $u \in S(x, y), z \in F(x, y, u)$, there exists $w \in F(x, y, v)$ such that $w \leq z$. Note that $v \in A(x, y)$. Thus, $A(x, y)$ is nonempty.

(II) For any $(x, y) \in E \times D, A(x, y)$ is closed subset of $E$.

In fact, letting $\left\{v_{\alpha}: \alpha \in I\right\} \subset A(x, y)$ with $v_{\alpha} \rightarrow v \in E$. Then $\left\{v_{\alpha}: \alpha \in I\right\} \subset$ $S(x, y)$. For any $u \in S(x, y)$ and $z \in F(x, y, u)$, there exists $w_{\alpha} \in F\left(x, y, v_{\alpha}\right)$ such that

$$
w_{\alpha} \leq z
$$

By the closedness of $S(x, y)$, it follows that $v \in S(x, y)$. If $v \notin A(x, y)$, then there exists $u_{0} \in S(x, y)$ and $z_{0} \in F\left(x, y, u_{0}\right)$ such that for any $w \in F(x, y, v), z_{0}-w \notin C$. Hence,

$$
\left(z_{0}-F(x, y, v)\right) \cap C=\emptyset .
$$

Since $C$ is closed convex cone and $F(x, y, v)$ is compact, there exists some neighborhood $U$ of 0 such that

$$
\left(z_{0}-(F(x, y, v)+U+C)\right) \cap C=\emptyset .
$$


Since $v_{\alpha} \rightarrow v$ and $F(x, y, v)$ is $C$-upper semicontinuous in $v$, there exists $\alpha_{0} \in I$ such that for all $\alpha \geq \alpha_{0}$,

$$
F\left(x, y, v_{\alpha}\right) \subset F(x, y, v)+U+C .
$$

By (3.9) and (3.10), we have

$$
\left(z_{0}-F\left(x, y, v_{\alpha}\right)\right) \cap C=\emptyset, \forall \alpha \geq \alpha_{0} .
$$

On the other hand, by (3.7), there exists $w_{\alpha} \in F\left(x, y, v_{\alpha}\right)$ such that $z_{0}-w_{\alpha} \in C$. This contradicts (3.11). Hence, $v \in A(x, y)$ and so $A(x, y)$ is closed.

(III) For any $(x, y) \in E \times D, A(x, y)$ is convex.

In fact, letting $v_{1}, v_{2} \in A(x, y)$, then $v_{1}, v_{2} \in S(x, y)$ and for any $u \in S(x, y)$, $z \in F(x, y, u)$, there exist $z_{1} \in F\left(x, y, v_{1}\right)$ and $z_{2} \in F\left(x, y, v_{2}\right)$ such that

$$
z_{1} \leq z \text { and } z_{2} \leq z
$$

By Lemma 2.3, $F(x, y, u)$ is $C$-quasiconvex in $u$. Thus, for any $t \in[0,1]$, there exists $z_{t} \in F\left(x, y, t v_{1}+(1-t) v_{2}\right)$ such that $z_{t} \leq z$. Since $S(x, y)$ is convex, we know that $t v_{1}+(1-t) v_{2} \in S(x, y)$ and so $t v_{1}+(1-t) v_{2} \in A(x, y)$. Hence, $A(x, y)$ is convex.

(IV) $A(x, y)$ is upper semicontinuous on $E \times D$.

By the compactness of $E \times D$, we only need to show that $A$ is a closed mapping. Let $\left\{\left(x_{\alpha}, y_{\alpha}\right): \alpha \in I\right\} \subset E \times D$ be a net such that $\left(x_{\alpha}, y_{\alpha}\right) \rightarrow(x, y) \in E \times D$. Let $v_{\alpha} \in A\left(x_{\alpha}, y_{\alpha}\right)$ with $v_{\alpha} \rightarrow v$. We will show $v \in A(x, y)$.

Since $S$ is upper semicontinuous mapping with nonempty closed values, it follows that $S$ is a closed mapping. Since $v_{\alpha} \in S\left(x_{\alpha}, y_{\alpha}\right)$ and $\left(x_{\alpha}, y_{\alpha}, v_{\alpha}\right) \rightarrow(x, y, v)$, then $v \in S(x, y)$. Next we show $v \in A(x, y)$, i.e.,

for any $u \in S(x, y), z \in F(x, y, u)$, there exists $w \in F(x, y, v)$, such that $w \leq z$.

If not, then there exists $u_{0} \in S(x, y)$ and $z_{0} \in F\left(x, y, u_{0}\right)$ such that for any $w \in$ $F(x, y, v), z_{0}-w \notin C$. Then

$$
\left(z_{0}-F(x, y, v)\right) \cap C=\emptyset .
$$

Since $C$ is closed convex cone and $F(x, y, v)$ is compact, there exists some neighborhood $U$ of 0 such that

$$
\left(z_{0}-(F(x, y, v)+U+C)\right) \cap C=\emptyset .
$$

There exists a balanced neighborhood $U_{1}$ of 0 such that $U_{1}-U_{1} \subset U$, and so

$$
\left(\left(z_{0}+U_{1}-C\right)-\left(F(x, y, v)+U_{1}+C\right)\right) \cap C=\emptyset .
$$


Since $u_{0} \in S(x, y),\left(x_{\alpha}, y_{\alpha}\right) \rightarrow(x, y), S$ is lower semicontinuous, there exists $u_{\alpha} \in$ $S\left(x_{\alpha}, y_{\alpha}\right)$ such that $u_{\alpha} \rightarrow u_{0}$. Hence, $\left(x_{\alpha}, y_{\alpha}, u_{\alpha}\right) \rightarrow\left(x, y, u_{0}\right)$ and $\left(x_{\alpha}, y_{\alpha}, v_{\alpha}\right) \rightarrow$ $(x, y, v)$. Since $F$ is $C$-lower semicontinuous and $C$-upper semicontinuous, for $z_{0} \in$ $F\left(x, y, u_{0}\right)$ and $U_{1}$, there exist neighborhood $U\left(x, y, u_{0}\right)$ of $\left(x, y, u_{0}\right)$ such that, when $\left(x^{\prime}, y^{\prime}, u^{\prime}\right) \in U\left(x, y, u_{0}\right)$,

$$
F\left(x^{\prime}, y^{\prime}, u^{\prime}\right) \cap\left(z_{0}+U_{1}-C\right) \neq \emptyset .
$$

Further, there exist neighborhood $U(x, y, v)$ of $(x, y, v)$ such that, when $\left(x^{\prime}, y^{\prime}, v^{\prime}\right) \in$ $U(x, y, v)$,

$$
F\left(x^{\prime}, y^{\prime}, v^{\prime}\right) \subset F(x, y, v)+U_{1}+C .
$$

Hence, there exists $\alpha_{0} \in I$ such that, when $\alpha \geq \alpha_{0}$,

$$
F\left(x_{\alpha}, y_{\alpha}, u_{\alpha}\right) \cap\left(z_{0}+U_{1}-C\right) \neq \emptyset .
$$

and

$$
F\left(x_{\alpha}, y_{\alpha}, v_{\alpha}\right) \subset F(x, y, v)+U_{1}+C .
$$

Let $z_{\alpha} \in F\left(x_{\alpha}, y_{\alpha}, u_{\alpha}\right) \cap\left(z_{0}+U_{1}-C\right)$. (3.13) and (3.15) implies that

$$
\left(\left(z_{\alpha}-\left(F\left(x_{\alpha}, y_{\alpha}, v_{\alpha}\right)\right) \cap C=\emptyset .\right.\right.
$$

Since $v_{\alpha} \in A\left(x_{\alpha}, y_{\alpha}\right)$, for $u_{\alpha} \in S\left(x_{\alpha}, y_{\alpha}\right)$ and $z_{\alpha} \in F\left(x_{\alpha}, y_{\alpha}, u_{\alpha}\right)$, there exists $w_{\alpha} \in F\left(x_{\alpha}, y_{\alpha}, v_{\alpha}\right)$ such that

$$
w_{\alpha} \leq z_{\alpha}
$$

This contradicts (3.16) and so $A$ is a closed mapping.

Similarly, we can prove that, for any $(x, y) \in E \times D, B$ is upper semicontinuous on $E \times D$ with nonempty closed convex values.

(V) Define the set-valued mapping $H: E \times D \rightarrow 2^{E \times D}$ by

$$
H(x, y)=(A(x, y), B(x, y)), \forall(x, y) \in E \times D .
$$

Then for each $(x, y) \in E \times D, H(x, y)$ is a nonempty closed convex subset of $E \times D$ and $H$ is upper semicontinuous on $E \times D$. By Lemma 2.2, there exists a point $(\bar{x}, \bar{y}) \in E \times D$ such that $(\bar{x}, \bar{y}) \in H(\bar{x}, \bar{y})$, i.e., $\bar{x} \in A(\bar{x}, \bar{y})$ and $\bar{y} \in B(\bar{x}, \bar{y})$. It follows from the definition of $A$ that for any $u \in S(\bar{x}, \bar{y})$ and $z \in F(\bar{x}, \bar{y}, u)$, there exists $w \in F(\bar{x}, \bar{y}, \bar{x})$ such that $w \leq z$. Similarly, for any $v \in T(\bar{x}, \bar{y})$ and $e \in G(\bar{y}, \bar{x}, v)$, there exists $d \in G(\bar{y}, \bar{x}, \bar{y})$ such that $d \leq e$. By (ii), we have $w \in C$ and $d \in C$. Thus, $z \in C$ and $e \in C$. It follows that $\bar{x} \in S(\bar{x}, \bar{y}), \bar{y} \in T(\bar{x}, \bar{y})$ and

$$
\begin{aligned}
& F(\bar{x}, \bar{y}, x) \subset C, \forall x \in S(\bar{x}, \bar{y}), \\
& G(\bar{y}, \bar{x}, y) \subset C, \forall y \in T(\bar{x}, \bar{y}) .
\end{aligned}
$$


Next we show that the solution set of GSSVQEP is closed. Let $\left\{\left(x_{\alpha}, y_{\alpha}\right): \alpha \in I\right\}$ be a net in the set of solutions of GSSVQEP such that $\left(x_{\alpha}, y_{\alpha}\right) \rightarrow(\bar{x}, \bar{y})$. Then $x_{\alpha} \in S\left(x_{\alpha}, y_{\alpha}\right), y_{\alpha} \in T\left(x_{\alpha}, y_{\alpha}\right)$ and

$$
\begin{aligned}
& F\left(x_{\alpha}, y_{\alpha}, x\right) \subset C, \forall x \in S\left(x_{\alpha}, y_{\alpha}\right), \\
& G\left(y_{\alpha}, x_{\alpha}, y\right) \subset C, \forall y \in T\left(x_{\alpha}, y_{\alpha}\right) .
\end{aligned}
$$

Since $S$ and $T$ are upper semicontinuous set-valued mappings with nonempty compact values, by Lemma 2.1, $S$ and $T$ are closed mappings and so $\bar{x} \in S(\bar{x}, \bar{y}), \bar{y} \in T(\bar{x}, \bar{y})$. Since $S$ and $T$ are lower semicontinuous set-valued mappings, for any $z \in S(\bar{x}, \bar{y})$, there exist $z_{\alpha} \in S\left(x_{\alpha}, y_{\alpha}\right)$ such that $z_{\alpha} \rightarrow z$. By (3.18), we have

$$
F\left(x_{\alpha}, y_{\alpha}, z_{\alpha}\right) \subset C \text {. }
$$

We claim that

$$
F(\bar{x}, \bar{y}, z) \subset C .
$$

If not, then there exists $m \in F(\bar{x}, \bar{y}, z)$ such that $m \notin C$. Thus, there exists some neighborhood $U$ of 0 such that $(m+U) \cap C=\emptyset$ and so

$$
(m+U-C) \cap C=\emptyset .
$$

Since $F: E \times D \times E \rightarrow 2^{Z}$ is $C$-lower semicontinuous mapping, there exists $\alpha_{0}$ such that when $\alpha \geq \alpha_{0}$,

$$
F\left(x_{\alpha}, y_{\alpha}, z_{\alpha}\right) \cap(m+U-C) \neq \emptyset .
$$

Taking $m_{\alpha} \in F\left(x_{\alpha}, y_{\alpha}, z_{\alpha}\right)$ and $m_{\alpha} \in(m+U-C)$, by (3.21), we have $m_{\alpha} \notin C$. However, by (3.20), we have $m_{\alpha} \in C$, which is a contradiction. Therefore, by the arbitrariness of $z \in S(\bar{x}, \bar{y})$, we have

$$
F(\bar{x}, \bar{y}, z) \subset C, \forall z \in S(\bar{x}, \bar{y}) .
$$

Similarly, we can prove that

$$
G(\bar{y}, \bar{x}, y) \subset C, \forall y \in T(\bar{x}, \bar{y}) .
$$

This shows that $(\bar{x}, \bar{y})$ is the solution of GSSVQEP and so the solution set of GSSVQEP is closed. This completes the proof.

Now we give an example to explain that Theorem 3.1 is applicable.

Example 3.1. Let $X=Y=Z=R, C=[0,+\infty)$, and $E=D=[0,1]$. For each $x \in E, y \in D, S(x, y)=[0,1]$ and $T(x, y)=[0,1]$. Define the set-valued mappings $F$ and $G$ as follows:

$$
F(x, y, z)=[2 x+y-z, 10], \forall(x, y, z) \in E \times D \times E,
$$




$$
G(y, x, z)=[x+2 y-z, 5], \forall(y, x, z) \in D \times E \times D .
$$

It is easy to check that all conditions in Theorem 3.1 are satisfied. Hence, by Theorem 3.1, GSSVQEP has a solution. Let $H$ be the solution set of GSSVQEP. Then,

$$
H=\{\bar{x}, \bar{y}) \in E \times D: 2 \bar{x}+\bar{y} \geq 1, \bar{x}+2 \bar{y} \geq 1\} .
$$

It is easy to see that $H$ is a closed subset of $E \times D$.

\section{STABility}

In this section, we discuss the stability of solutions for generalized symmetric strong vector quasi-equilibrium problems.

Let $(X, d)$ be a metric space. Denote by $K(X), B C(X)$, and $C K(X)$ all nonempty compact subsets of $X$, all nonempty bounded closed subsets of $X$, and all nonempty convex compact subsets of $X$ (if $X$ is a linear metric space), respectively. Let $B_{1}, B_{2} \subset$ $X$ and define

$$
h\left(B_{1}, B_{2}\right)=\max \left\{h^{0}\left(B_{1}, B_{2}\right), h^{0}\left(B_{2}, B_{1}\right)\right\},
$$

where $h^{0}\left(B_{1}, B_{2}\right)=\sup _{b \in B_{1}} d\left(b, B_{2}\right)$, and $d\left(b, B_{2}\right)=\inf _{b^{\prime} \in B_{2}} d\left(b, b^{\prime}\right)$. It is obvious that $h$ is a Hausdorff metric on $K(X), B C(X), C K(X)$ respectively.

Lemma 4.1. ([32]). Let $E$ be a nonempty compact subset of $\left(X,\|\cdot\|_{X}\right)$ and $D$ be a nonempty compact subset of $\left(Y,\|\cdot\|_{Y}\right)$. Let $S: E \times D \rightarrow 2^{E}$ and $T: E \times D \rightarrow 2^{D}$ be two set-valued mappings with nonempty compact values. Then $S$ and $T$ are continuous if and only if, for any $\left(x^{*}, y^{*}\right) \in E \times D,(x, y) \rightarrow\left(x^{*}, y^{*}\right)$, implies

$$
S(x, y) \stackrel{h}{\longrightarrow} S\left(x^{*}, y^{*}\right) \text { and } T(x, y) \stackrel{h^{\prime}}{\longrightarrow} T\left(x^{*}, y^{*}\right),
$$

where $h$ is a Hausdorff metric on $K(E)$ and $h^{\prime}$ is a Hausdorff metric on $K(D)$.

Lemma 4.2. ([32]). Let $(X, d)$ be a metric space and $h$ be Hausdorff metric on $X$. Then

(i) $(B C(X), h)$ is complete if and only if $(X, d)$ is complete;

(ii) $(K(X), h)$ is complete if and only if $(X, d)$ is complete;

(iii) If $X$ is a linear metric space, then $(C K(X), h)$ is complete if and only if $(X, d)$ is complete.

Lemma 4.3. ([36]). Let $W$ be a metric space, $A, A_{n} \subset W(n=1,2, \cdots)$ be compact subsets. If for any open set $O$ with $A \subset O$, there exists $n_{0}$ such that $A_{n} \subset O$ for all $n \geq n_{0}$, then any sequence $\left\{x_{n}\right\}$, satisfying $x_{n} \in A_{n}$ has a convergent subsequence with limit in A. 
Throughout this section, let $X, Y$ and $Z$ be Banach spaces. Let $E \subset X$ and $D \subset Y$ be nonempty compact convex sets. Let

$M=\left\{(S, T, F, G): S: E \times D \rightarrow 2^{E}\right.$ and $T: E \times D \rightarrow 2^{D}$ are continuous set-valued mappings with nonempty compact convex values, $F: E \times D \times E \rightarrow 2^{Z}$ and $G$ : $D \times E \times D \rightarrow 2^{Z}$ are $C$-upper semicontinuous and $C$-lower semicontinuous mappingswith nonempty compact values such that for every fixed $(x, y) \in E$ $\times D, F(x, y, u)$ is $C$-properly quasiconvex in $u$ and $G(y, x, v)$ is $C$-properly quasiconvex in $v$ \}.

For any $u_{1}=\left(S_{1}, T_{1}, F_{1}, G_{1}\right)$ and $u_{2}=\left(S_{2}, T_{2}, F_{2}, G_{2}\right) \in M$, define

$$
\begin{aligned}
\rho\left(u_{1}, u_{2}\right)= & \sup _{(x, y) \in E \times D} h_{1}\left(S_{1}(x, y), S_{2}(x, y)\right)+\sup _{(x, y) \in E \times D} h_{2}\left(T_{1}(x, y), T_{2}(x, y)\right) \\
& +\sup _{(x, y, u) \in E \times D \times E} h_{3}\left(F_{1}(x, y, u), F_{2}(x, y, u)\right) \\
& +\sup _{(y, x, v) \in D \times E \times D} h_{3}\left(G_{1}(y, x, v), G_{2}(y, x, v)\right) .
\end{aligned}
$$

where $h_{1}, h_{2}$ and $h_{3}$ are Hausdorff metrics on $C K(E), C K(D)$ and $C(Z)$, respectively.

Proposition 4.1. $(M, \rho)$ is a complete metric space.

Proof. It is clear that $(M, \rho)$ is a metric space. Now, we show that $(M, \rho)$ is complete.

Let $\left\{u_{n}\right\}$ be any Cauchy sequence in $M$, where $u_{n}=\left(S_{n}, T_{n}, F_{n}, G_{n}\right), n=1,2, \ldots$ Then, for any $\varepsilon>0$, there exists $N$ such that

$$
\rho\left(u_{n}, u_{m}\right)<\varepsilon / 4, \forall n, m \geq N .
$$

It follows that, for any $(x, y, u, v) \in E \times D \times E \times D$,

$$
h_{1}\left(S_{n}(x, y), S_{m}(x, y)\right)<\varepsilon / 4, \quad h_{2}\left(T_{n}(x, y), T_{m}(x, y)\right)<\varepsilon / 4,
$$

and

$$
h_{3}\left(F_{n}(x, y, u), F_{m}(x, y, u)\right)<\varepsilon / 4, \quad h_{3}\left(G_{n}(y, x, v), G_{m}(y, x, v)\right)<\varepsilon / 4
$$

Then, for any fixed $(x, y, u, v) \in E \times D \times E \times D,\left\{S_{n}(x, y)\right\}$ is a Cauchy sequence in $C K(E),\left\{T_{n}(x, y)\right\}$ is a Cauchy sequence in $C K(D)$, and $\left\{F_{n}(x, y, u)\right\}$, $\left\{G_{n}(y, x, v)\right\}$ are two Cauchy sequences in $K(Z)$. By Lemma 4.2 and assumption, $\left(C K(E), h_{1}\right),\left(C K(D), h_{2}\right)$ and $\left(K(Z), h_{3}\right)$ are complete spaces. It follows 
that there exist $S(x, y) \in C K(E), T(x, y) \in C K(D), F(x, y, u) \in K(Z)$ and $G(y, x, v) \in K(Z)$ such that

$$
S_{n}(x, y) \stackrel{h_{1}}{\longrightarrow} S(x, y), \quad T_{n}(x, y) \stackrel{h_{2}}{\longrightarrow} T(x, y),
$$

and

$$
F_{n}(x, y, u) \stackrel{h_{3}}{\longrightarrow} F(x, y, u), \quad G_{n}(y, x, v) \stackrel{h_{3}}{\longrightarrow} G(y, x, v) .
$$

Since $h_{1}(\cdot, \cdot), h_{2}(\cdot, \cdot)$ and $h_{3}(\cdot, \cdot)$ are continuous, by (4.2) and (4.3), for any fixed $n \geq N$ and any $(x, y, u, v) \in E \times D \times E \times D$, letting $m \rightarrow \infty$, we get

$$
h_{1}\left(S_{n}(x, y), S(x, y)\right) \leq \varepsilon / 4, \quad h_{2}\left(T_{n}(x, y), T(x, y)\right) \leq \varepsilon / 4,
$$

and

$$
h_{3}\left(F_{n}(x, y, u), F(x, y, u)\right) \leq \varepsilon / 4, \quad h_{3}\left(G_{n}(y, x, v), G(y, x, v)\right) \leq \varepsilon / 4
$$

Now we show that $S$ is continuous.

By Lemma 4.1, we need to prove that, for any fixed $\left(x_{0}, y_{0}\right) \in E \times D$ and any $\varepsilon>0$, there exists a neighborhood $N\left(x_{0}, y_{0}\right)$ of $\left(x_{0}, y_{0}\right)$ in $E \times D$ such that

$$
h_{1}\left(S(x, y), S\left(x_{0}, y_{0}\right)\right)<\varepsilon, \forall(x, y) \in N\left(x_{0}, y_{0}\right) \cap E \times D .
$$

Since

$$
\begin{aligned}
& h_{1}\left(S(x, y), S\left(x_{0}, y_{0}\right)\right) \\
\leq & h_{1}\left(S(x, y), S_{n}(x, y)\right)+h_{1}\left(S_{n}(x, y), S_{n}\left(x_{0}, y_{0}\right)\right) \\
& +h_{1}\left(S_{n}\left(x_{0}, y_{0}\right), S\left(x_{0}, y_{0}\right)\right),
\end{aligned}
$$

by (4.6), there exists $N$ such that, for any $n>N$,

$$
h_{1}\left(S(x, y), S_{n}(x, y)\right) \leq \varepsilon / 4, \forall(x, y) \in E \times D
$$

Taking a fixed $n>N$, by the continuity of $S_{n}$ and Lemma 4.1, there exists a neighborhood $N\left(x_{0}, y_{0}\right)$ of $\left(x_{0}, y_{0}\right)$ in $E \times D$ such that

$$
h_{1}\left(S_{n}(x, y), S_{n}\left(x_{0}, y_{0}\right)\right)<\varepsilon / 4, \forall(x, y) \in N\left(x_{0}, y_{0}\right) \cap E \times D .
$$

By (4.6), (4.8)-(4.10), we have

$$
h_{1}\left(S(x, y), S\left(x_{0}, y_{0}\right)\right)<\varepsilon, \forall(x, y) \in N\left(x_{0}, y_{0}\right) \cap E \times D .
$$

Hence, $S$ is continuous on $E \times D$. 
Similarly, we can prove $T$ is continuous on $E \times D$.

Now we show that $F$ is $C$-upper semicontinuous.

Pick any $\left(x_{0}, y_{0}, u_{0}\right) \in E \times D \times E$. For any neighborhood $V$ of 0 in $Z$, there exists $r>0$ such that

$$
r B_{Z} \subset V,
$$

where $B_{Z}$ is the open unit ball of $Z$. Taking a fixed $n_{0} \geq N$, by (4.7), we have

$$
h_{3}\left(F_{n_{0}}\left(x_{0}, y_{0}, u_{0}\right), F\left(x_{0}, y_{0}, u_{0}\right)\right) \leq \varepsilon / 4 .
$$

By the $C$-upper semicontinuity of $F_{n_{0}}$, there exists some neighborhood $U\left(x_{0}, y_{0}, u_{0}\right)$ of $\left(x_{0}, y_{0}, u_{0}\right)$ such that

$$
\begin{aligned}
& F_{n_{0}}(x, y, u) \subset F_{n_{0}}\left(x_{0}, y_{0}, u_{0}\right) \\
& +{ }^{\varepsilon} /{ }_{4} B_{Z}+C, \forall(x, y, u) \in U\left(x_{0}, y_{0}, u_{0}\right) \cap E \times D \times E
\end{aligned}
$$

It follows from (4.7) that, we have

(4.14) $F(x, y, u) \subset F_{n_{0}}(x, y, u)+{ }^{\varepsilon} / 4 \bar{B}_{Z}, \forall(x, y, u) \in U\left(x_{0}, y_{0}, u_{0}\right) \cap E \times D \times E$,

where $\bar{B}_{Z}$ is the closed unit ball of $Z$. From (4.12), (4.13) and (4.14), we get

$$
\begin{aligned}
F(x, y, u) & \subset F_{n_{0}}(x, y, u)+\varepsilon / 4 \bar{B}_{Z} \\
& \subset F_{n_{0}}\left(x_{0}, y_{0}, u_{0}\right)+\varepsilon / 4 B_{Z}+\varepsilon / 4 \bar{B}_{Z}+C \\
& \subset F\left(x_{0}, y_{0}, u_{0}\right)+\varepsilon / 4 \bar{B}_{Z}+\varepsilon / 4 B_{Z}+\varepsilon / 4 \bar{B}_{Z}+C \\
& \subset F\left(x_{0}, y_{0}, u_{0}\right)+3 \varepsilon / 4 \bar{B}_{Z}+C .
\end{aligned}
$$

Due to the arbitrariness of $\varepsilon$, we can pick $\varepsilon$ such that $3 \varepsilon / 4<r$. By (4.11) and (4.15), for all $(x, y, u) \in U\left(x_{0}, y_{0}, u_{0}\right) \cap E \times D \times E$, we have

$$
F(x, y, u) \subset F\left(x_{0}, y_{0}, u_{0}\right)+r B_{Z}+C \subset F\left(x_{0}, y_{0}, u_{0}\right)+V+C,
$$

and so $F$ is $C$-upper semicontinuous on $\left(x_{0}, y_{0}, u_{0}\right)$. Since $\left(x_{0}, y_{0}, u_{0}\right)$ is arbitrary, $F$ is $C$-upper semicontinuous on $E \times D \times E$.

Next we show that $F$ is $C$-lower semicontinuous.

Pick any $\left(x_{1}, y_{1}, u_{1}\right) \in E \times D \times E$. For any $z \in F\left(x_{1}, y_{1}, u_{1}\right)$ and for any neighborhood $V$ of 0 in $Z$, there exists $t>0$ such that

$$
t B_{Z} \subset V .
$$

Taking a fixed $n_{1} \geq N$, by (4.7), we have

$$
h_{3}\left(F_{n_{1}}\left(x_{1}, y_{1}, u_{1}\right), F\left(x_{1}, y_{1}, u_{1}\right)\right) \leq \varepsilon / 4 \text {. }
$$


Since $F_{n_{1}}\left(x_{1}, y_{1}, u_{1}\right)$ and $F\left(x_{1}, y_{1}, u_{1}\right)$ are compact sets, there exists $z_{n_{1}} \in F_{n_{1}}\left(x_{1}\right.$, $\left.y_{1}, u_{1}\right)$ such that

$$
\left\|z_{n_{1}}-z\right\| \leq h_{3}\left(F_{n_{1}}\left(x_{1}, y_{1}, u_{1}\right), F\left(x_{1}, y_{1}, u_{1}\right)\right) \leq \varepsilon / 4 .
$$

Since $F_{n_{1}}$ is $C$-lower semicontinuous, there exists some neighborhood $U\left(x_{1}, y_{1}, u_{1}\right)$ of $\left(x_{1}, y_{1}, u_{1}\right)$ such that

$$
\begin{aligned}
& F_{n_{1}}(x, y, u) \cap\left(z_{n_{1}}+\varepsilon /{ }_{4} B_{Z}-C\right) \\
\neq & \emptyset, \forall(x, y, u) \in U\left(x_{1}, y_{1}, u_{1}\right) \cap E \times D \times E .
\end{aligned}
$$

By the arbitrariness of $\varepsilon$, we can pick $\varepsilon$ such that $3 \varepsilon / 4<t$. We claim that

$$
\begin{aligned}
& F(x, y, u) \cap\left(z+t B_{Z}-C\right) \\
\neq & \emptyset, \forall(x, y, u) \in U\left(x_{1}, y_{1}, u_{1}\right) \cap E \times D \times E .
\end{aligned}
$$

Indeed, by (4.19), there exists $d \in F_{n_{1}}(x, y, u)$ and $d \in z_{n_{1}}+{ }^{\varepsilon} /{ }_{4} B_{Z}-C$. It follows from (4.7) that

$$
F_{n_{1}}(x, y, u) \subset F(x, y, u)+{ }^{\varepsilon} / 4 \bar{B}_{Z},
$$

Hence, for $d \in F_{n_{1}}(x, y, u)$, there exists $a \in F(x, y, u)$ and $b \in \varepsilon / 4 \bar{B}_{Z}$ such that $d=a+b$. By (4.18),

$$
\begin{aligned}
a=d-b & \in z_{n_{1}}+{ }^{\varepsilon} / 4 B_{Z}-C-\varepsilon / 4 \bar{B}_{Z} \\
& \subset z+{ }^{\varepsilon} / 4 \bar{B}_{Z}+{ }^{\varepsilon} / 4 B_{Z}-C-\varepsilon / 4 \bar{B}_{Z} \\
& \subset z+3 \varepsilon / 4 \bar{B}_{Z}-C \\
& \subset z+t B_{Z}-C .
\end{aligned}
$$

and so

$$
F(x, y, u) \cap\left(z+t B_{Z}-C\right) \neq \emptyset .
$$

From (4.16) and (4.23), we get

$$
F(x, y, u) \cap(z+V-C) \neq \emptyset, \forall(x, y, u) \in U\left(x_{1}, y_{1}, u_{1}\right) \cap E \times D \times E .
$$

Hence, $F$ is $C$-lower semicontinuous on $\left(x_{1}, y_{1}, u_{1}\right)$. Since $\left(x_{1}, y_{1}, u_{1}\right)$ is arbitrary, $F$ is $C$-lower semicontinuous on $E \times D \times E$.

Next we show that, for any fixed $(x, y) \in E \times D, F(x, y, u)$ is $C$-properly quasiconvex in $u$. 
Indeed, for any $u_{1}, u_{2} \in E, z_{1} \in F\left(x, y, u_{1}\right), z_{2} \in F\left(x, y, u_{2}\right), t \in[0,1]$, since $F\left(x, y, u_{1}\right), F\left(x, y, u_{2}\right), F_{n}\left(x, y, u_{1}\right)$ and $F_{n}\left(x, y, u_{2}\right)$ are compact sets, by (4.7), there exist $a_{n} \in F_{n}\left(x, y, u_{1}\right), b_{n} \in F_{n}\left(x, y, u_{2}\right)$ such that

$$
\left\|a_{n}-z_{1}\right\| \leq h_{3}\left(F_{n}\left(x, y, u_{1}\right), F\left(x, y, u_{1}\right)\right) \leq \varepsilon / 4
$$

and

$$
\left\|b_{n}-z_{2}\right\| \leq h_{3}\left(F_{n}\left(x, y, u_{2}\right), F\left(x, y, u_{2}\right)\right) \leq \varepsilon / 4 .
$$

By the $C$-properly quasiconvexity of $F_{n}$, there exists $c_{n} \in F_{n}\left(x, y, t u_{1}+(1-t) u_{2}\right)$ such that

$$
\text { either } c_{n} \leq a_{n} \text { or } c_{n} \leq b_{n},
$$

Since $F_{n}\left(x, y, t u_{1}+(1-t) u_{2}\right)$ and $F\left(x, y, t u_{1}+(1-t) u_{2}\right)$ are compact sets, by (4.7), there exists $d_{n} \in F\left(x, y, t u_{1}+(1-t) u_{2}\right)$ such that

$$
\begin{aligned}
& \left\|c_{n}-d_{n}\right\| \\
& \leq h_{3}\left(F_{n}\left(x, y, t u_{1}+(1-t) u_{2}\right), F\left(x, y, t u_{1}+(1-t) u_{2}\right)\right) \leq \varepsilon / 4 .
\end{aligned}
$$

By the compactness of $F\left(x, y, t u_{1}+(1-t) u_{2}\right)$, there exist a subsequence $\left\{d_{n_{k}}\right\}$ of $\left\{d_{n}\right\}$ such that $d_{n_{k}} \rightarrow d \in F\left(x, y, t u_{1}+(1-t) u_{2}\right)$. Hence, by (4.27), we have $c_{n_{k}} \rightarrow d$. From (4.24), (4.25) and (4.26), we have

$$
\text { either } d \leq z_{1} \text { or } d \leq z_{2} \text {. }
$$

Hence, $F(x, y, u)$ is $C$-properly quasiconvex in $u$.

Similarly, we can prove that $G$ is $C$-upper semicontinuous and $C$-lower semicontinuous mappings with nonempty compact values. For every fixed $(x, y) \in E \times D$, $G(y, x, v)$ is $C$-properly quasiconvex in $v$. By (4.6) and (4.7), for any fixed $n \geq N$ and any $(x, y, u, v) \in E \times D \times E \times D$, we have

$$
\begin{gathered}
\sup _{(x, y) \in E \times D} h_{1}\left(S_{n}(x, y), S(x, y)\right) \leq \varepsilon / 4, \sup _{(x, y) \in E \times D} h_{2}\left(T_{n}(x, y), T(x, y)\right) \leq \varepsilon / 4, \\
\sup _{(x, y, u) \in E \times D \times E} h_{3}\left(F_{n}(x, y, u), F(x, y, u)\right) \leq \varepsilon / 4, \sup _{(y, x, v) \in D \times E \times D} h_{3}\left(G_{n}(y, x, v),\right. \\
G(y, x, v)) \leq \varepsilon / 4 .
\end{gathered}
$$

Set $u=(S, T, F, G)$. We know that $u \in M$ and $\rho\left(u_{n}, u\right) \leq \varepsilon$ for all $n \geq N$, i.e., $u_{n} \stackrel{\rho}{\longrightarrow} u$. Hence, $(M, \rho)$ is a complete metric space. This completes the proof.

For any $u=(S, T, F, G) \in M$, Theorem 3.1 implies that GSSVQEP has a solution. Denote by $\psi(u)$ the solution set of GSSVQEP respect to $u$. Then, $\psi$ defines a setvalued mapping from $\mathrm{M}$ to $C \times D$ and $\psi(u) \neq \emptyset$ for each $u \in M$. 
Theorem 4.2. $\psi: M \rightarrow 2^{E \times D}$ is a upper semicontinuous mapping with compact values.

Proof. Since $E \times D$ is compact, it suffices to prove that $\psi$ is a closed mapping. Let a sequence $\left\{\left(u_{n},\left(x_{n}, y_{n}\right)\right)\right\} \subset \operatorname{Graph}(\psi)$ be given such that $\left(u_{n},\left(x_{n}, y_{n}\right)\right) \rightarrow$ $(u,(\bar{x}, \bar{y})) \in M \times(E \times D)$, where $u_{n}=\left(S_{n}, T_{n}, F_{n}, G_{n}\right)$ and $u=(S, T, F, G)$.

For each $n$, since $\left(x_{n}, y_{n}\right) \in \psi\left(u_{n}\right)$, we have

$$
x_{n} \in S_{n}\left(x_{n}, y_{n}\right), y_{n} \in T_{n}\left(x_{n}, y_{n}\right),
$$

$$
F_{n}\left(x_{n}, y_{n}, x\right) \subset C, \forall x \in S_{n}\left(x_{n}, y_{n}\right),
$$

and

$$
G_{n}\left(y_{n}, x_{n}, y\right) \subset C, \forall y \in T_{n}\left(x_{n}, y_{n}\right),
$$

For any open set $O$ with $S(\bar{x}, \bar{y}) \subset O$, since $S(\bar{x}, \bar{y})$ is a compact set, there exists $\varepsilon>0$ such that

$$
\{x \in E: d(x, S(\bar{x}, \bar{y}))<\varepsilon\} \subset O,
$$

where $d(x, S(\bar{x}, \bar{y}))=\inf _{x^{\prime} \in S(\bar{x}, \bar{y})}\left\|x-x^{\prime}\right\|$. Since $\rho\left(\left(S_{n}, T_{n}, F_{n}, G_{n}\right),(S, T, F, G)\right)$ $\rightarrow 0,\left(x_{n}, y_{n}\right) \rightarrow(\bar{x}, \bar{y})$ and $S$ is upper semicontinuous at $(\bar{x}, \bar{y})$, there exists $N$ such that, for any $n \geq N$,

$$
\sup _{(x, y) \in E \times D} h_{1}\left(S_{n}(x, y), S(x, y)\right)<\frac{1}{2} \varepsilon
$$

and

$$
S\left(x_{n}, y_{n}\right) \subset\left\{x \in E: d(x, S(\bar{x}, \bar{y}))<\frac{1}{2} \varepsilon\right\} .
$$

From (4.31), (4.32) and (4.33), we have

$$
\begin{aligned}
S_{n}\left(x_{n}, y_{n}\right) & \subset\left\{x \in E: d\left(x, S\left(x_{n}, y_{n}\right)\right)<\frac{1}{2} \varepsilon\right\} \\
& \subset\{x \in E: d(x, S(\bar{x}, \bar{y}))<\varepsilon\} \subset O, \forall n \geq N .
\end{aligned}
$$

Noting that $S(\bar{x}, \bar{y}) \subset O,(4.34)$ and $x_{n} \in S_{n}\left(x_{n}, y_{n}\right)$, we can apply Lemma 4.3 to get a subsequence $\left\{x_{n_{k}}\right\}$ of $\left\{x_{n}\right\}$ which converges to $\bar{x}_{0} \in S(\bar{x}, \bar{y})$. Since $\left\{x_{n_{k}}\right\} \rightarrow \bar{x}$,

$$
\bar{x}=x_{0} \in S(\bar{x}, \bar{y}) .
$$




\section{Similarly}

$$
\bar{y} \in T(\bar{x}, \bar{y}) .
$$

From the lower semicontinuity of $S$ at $(\bar{x}, \bar{y})$ and $\left(x_{n}, y_{n}\right) \rightarrow(\bar{x}, \bar{y})$, for any $x \in$ $S(\bar{x}, \bar{y})$, there exists $z_{n} \in S\left(x_{n}, y_{n}\right)$ such that $z_{n} \rightarrow x$. Since $\rho\left(\left(S_{n}, T_{n}, F_{n}, G_{n}\right)\right.$, $(S, T, F, G)) \rightarrow 0$, we can choose a subsequence $\left\{S_{n_{k}}\right\}$ of $\left\{S_{n}\right\}$ such that

$$
\sup _{(x, y) \in E \times D} h_{1}\left(S_{n_{k}}(x, y), S(x, y)\right)<\frac{1}{k} .
$$

Thus,

$$
h_{1}\left(S_{n_{k}}\left(x_{n_{k}}, y_{n_{k}}\right), S\left(x_{n_{k}}, y_{n_{k}}\right)\right)<\frac{1}{k} .
$$

This implies that there exist $z_{n_{k}}^{\prime} \in S_{n_{k}}\left(x_{n_{k}}, y_{n_{k}}\right)$ such that

$$
\left\|z_{n_{k}}^{\prime}-z_{n_{k}}\right\|<\frac{1}{k}
$$

Since

$$
\left\|z_{n_{k}}^{\prime}-x\right\| \leq\left\|z_{n_{k}}^{\prime}-z_{n_{k}}\right\|+\left\|z_{n_{k}}-x\right\|<\frac{1}{k}+\left\|z_{n_{k}}-x\right\| \rightarrow 0,
$$

we have $z_{n_{k}}^{\prime} \rightarrow x$. Since $z_{n_{k}}^{\prime} \in S_{n_{k}}\left(x_{n_{k}}, y_{n_{k}}\right), x_{n_{k}} \in S_{n_{k}}\left(x_{n_{k}}, y_{n_{k}}\right)$ and $y_{n_{k}} \in$ $T_{n_{k}}\left(x_{n_{k}}, y_{n_{k}}\right)$, by (4.29), we have

$$
F_{n_{k}}\left(x_{n_{k}}, y_{n_{k}}, z_{n_{k}}^{\prime}\right) \subset C .
$$

We claim that

$$
F(\bar{x}, \bar{y}, x) \subset C .
$$

If not, then there exists $z \in F(\bar{x}, \bar{y}, x)$ such that $z \notin C$. Hence, there exists some neighborhood $U$ of 0 in $Z$ such that $(z+U) \cap C=\emptyset$ and so

$$
(z+U-C) \cap C=\emptyset .
$$

Thus, there exists $r>0$ such that $r B_{z} \subset U$. Since $F$ is $C$-lower semicontinuous mapping, when $k$ is sufficiently large, we have

$$
F\left(x_{n_{k}}, y_{n_{k}}, z_{n_{k}}^{\prime}\right) \cap\left(z+\frac{r}{3} B_{Z}-C\right) \neq \emptyset .
$$

Let $s_{n_{k}} \in F\left(x_{n_{k}}, y_{n_{k}}, z_{n_{k}}^{\prime}\right) \cap\left(z+\frac{r}{3} B_{Z}-C\right)$. Since $\rho\left(\left(S_{n}, T_{n}, F_{n}, G_{n}\right),(S, T\right.$, $F, G)) \rightarrow 0$, for any $\varepsilon>0\left(\varepsilon<\frac{r}{3}\right)$, there exists $N$ such that, for any $n \geq N$,

$$
\sup _{(x, y, u) \in E \times D \times E} h_{3}\left(F_{n}(x, y, u), F(x, y, u)\right) \leq \varepsilon .
$$


When $k$ is sufficient large such that $n_{k} \geq N$, by (4.39), we have

$$
\sup _{(x, y, u) \in E \times D \times E} h_{3}\left(F_{n_{k}}(x, y, u), F(x, y, u)\right) \leq \varepsilon
$$

and so

$$
h_{3}\left(F_{n_{k}}\left(x_{n_{k}}, y_{n_{k}}, z_{n_{k}}^{\prime}\right), F\left(x_{n_{k}}, y_{n_{k}}, z_{n_{k}}^{\prime}\right)\right) \leq \varepsilon .
$$

For $s_{n_{k}} \in F\left(x_{n_{k}}, y_{n_{k}}, z_{n_{k}}^{\prime}\right)$, by (4.40), there exists $t_{n_{k}} \in F_{n_{k}}\left(x_{n_{k}}, y_{n_{k}}, z_{n_{k}}^{\prime}\right)$ such that

$$
\left\|s_{n_{k}}-t_{n_{k}}\right\| \leq \varepsilon
$$

and so

$$
t_{n_{k}} \in s_{n_{k}}+\varepsilon \bar{B}_{Z} \subset z+\frac{r}{3} B_{Z}-C+\varepsilon \bar{B}_{Z} \subset z+r B_{Z}-C \subset z+U-C .
$$

From (4.37), we have

$$
t_{n_{k}} \in C \cap(z+U-C),
$$

which contradicts (4.38). By the arbitrariness of $x \in S(\bar{x}, \bar{y})$, we have

$$
F(\bar{x}, \bar{y}, x) \subset C, \forall x \in S(\bar{x}, \bar{y}),
$$

Similarly, we can prove that

$$
G(\bar{y}, \bar{x}, y) \subset C, \forall y \in T(\bar{x}, \bar{y}) .
$$

By (4.35), (4.36), (4.41) and (4.42), we know that $((S, T, F, G),(\bar{x}, \bar{y})) \in \operatorname{Graph}(\psi)$. Hence, $\operatorname{Graph}(\psi)$ is closed. By Theorem 3.1, we know $\psi(u)$ is closed. By the compactness of $E \times D$, it is easy to see that $\psi(u) \subset E \times D$ is compact. Therefore, $\psi$ is a upper semicontinuous mapping with compact values. This completes the proof.

\section{REFERENCES}

1. J. P. Aubin and I. Ekeland, Applied Nonlinear Analysis, John Wiley, New York, 1984.

2. L. Q. Anh and P. Q. Khanh, Existence conditions in symmetric multivalued vector quasiequilibrium problems, Control. Cyber., 36 (2007), 519-530.

3. L. Q. Anh and P. Q. Khanh, Various kinds of semicontinuity and the solution sets of parametric multivalued symmetric vector quasiequilibrium problems, J. Glob. Optim., 41 (2008), 539-558.

4. Q. H. Ansari, Existence of solutions of systems of generalized implicit vector quasiequilibrium problems, J. Math. Anal. Appl., 341 (2008), 1271-1283. 
5. Q. H. Ansari, I. V. Konnov and J. C. Yao, Existence of a solution and variational principles for vector equilibrium problems, J. Optim. Theory. Appl., 110 (2001), 481492.

6. Q. H. Ansari and G. M. Lee, Nonsooth vector optimization problems and Minty vector variational inequalities, J. Optim. Theory. Appl., 145 (2010), 1-16.

7. E. Blum and W. Oettli, From optimization and variational inequalities to equilibrium problems, Math. Stud., 63 (1994), 123-145.

8. B. Chen, X. H. Gong and S. M. Yuan, Connectedness and compactness of weak efficient solutions for set-Valued vector equilibrium problems, J. Inequal. Appl., 83 (2008), 83-96.

9. C. R. Chen, S. J. Li and Z. M. Fang, On the solution semicontinuity to a parametric generalized vector quasivariational inequality, Comput. Math. Appl., 60 (2010), 24172425 .

10. G. Y. Chen, Existence of solutions for a vector variational inequality: an extension of Hartman-Stampacchia theorem, J. Optim. Theory. Appl., 74 (1992), 445-456.

11. G. Y. Chen, X. X. Huang and X. Q. Yang, Vector optimization: Set-Valued and Variational Analysis, Vol. 541, Lecture Notes in Economics and Mathematical Systems, Springer, Berlin, Germany, 2005.

12. J. C. Chen and X. H. Gong, The stability of set of solutions for symmetric vector quasiequilibrium problems, J. Optim. Theory. Appl., 136 (2008), 359-374.

13. T. D. Chuong and J. C. Yao, Generalized Clarke epiderivatives of parametric vector optimization problems, J. Optim. Theory. Appl., 146 (2010), 77-94.

14. M. Fakhar and J. Zafarani, Generalized symmetric vector quasiequilibrium problems, $J$. Optim. Theory. Appl., 136 (2008), 397-409.

15. K. Fan, A generalization of Tychonoff's fixed point theorem, Math. Ann., 142 (1961), 303-310.

16. A. P. Farajzadeh, On the symmetric vector quasiequilibrium problems, J. Math. Anal. Appl., 322 (2006), 1099-1110.

17. Y. P. Fang and N. J. Huang, Feasibility and solvability of vector variational inequalities with moving cones in Banach spaces, Nonlinear Anal., 70 (2009), 2024-2034.

18. F. Ferro, Optimization and stability results through cone lower semicontinuity, Set-valued. Anal., 5 (1997), 365-375.

19. J. Y. Fu, Symmetric vector quasiequilibrium problems, J. Math. Anal. Appl., 285 (2003), 708-713.

20. F. Giannessi, Theorems of the alternative quadratic programs and complementarity problems, in: Variational Inequalities and Complementarity Problems, (R. W. Cottle, F. Giannessi and J. L. Lions, eds.), Wiley, Chichester, 1980, pp. 151-186.

21. F. Giannessi, On Minty Variational Principle, in: New Trends in Mathematical Programming, Kluwer Academic Publishers, Dordrecht, Netherlands, 1998. 
22. X. H. Gong, Continuity of the solution set to parametric weak vector equilibrium problems, J. Optim. Theory. Appl., 139 (2008), 35-46.

23. X. H. Gong, Symmetric strong vector quasi-equilibrium problems, Math. Methods. Oper. Res., 65 (2007), 305-314.

24. X. H. Gong, Strong vector equilibrium problems, J. Global. Optim., 36 (2006), 339-349.

25. R. B. Holmes, Geometric Function Analysis and its Application, Springer-Verlag, New York, 1975.

26. S. H. Hou, X. H. Gong and X. M. Yang, Existence and stability of solutions for generalized strong vector equilibrium problems with trifunctions, J. Optim. Theory. Appl., 146 (2010), 387-398.

27. N. J. Huang and Y. P. Fang, On vector variational-like inequalities in reflexive Banach spaces, J. Glob. Optim., 32 (2005), 495-505.

28. N. J. Huang, J. Li and J. C. Yao, Gap functions and existence of solutions to a system of vector equilibrium problems., J. Optim. Theory. Appl., 133 (2007), 201-212.

29. P. Q. Khanh and L. M. Luu, Upper semicontinuity of the solution set to parametric vector quasivariational inequalities, J. Global. Optim., 32 (2005), 569-580.

30. K. Kimura and J. C. Yao, Sensitivity analysis of solution mappings of parametric vector quasiequilibrium problems, J. Global. Optim., 41 (2008), 187-202.

31. K. Kimura and J. C. Yao, Semicontinuity of solution mappings of parametric generalized vector equilibrium problems, J. Optim. Theory. Appl., 138 (2008), 429-443.

32. E. Klein and A. C. Thompson, Theory of Correspondences, Wiley, New York, 1984.

33. X. J. Long, N. J. Huang and K. L. Teo, Existence and stability of solutions for generalized strong vector quasi-equilibrium problem, Math. Comput. Model., 47 (2008), 445-451.

34. M. A. Noor and W. Oettli, On general nonlinear complementarity problems and quasiequilibria, Mathmatiche XLIX, 1994, pp. 313-331.

35. J. C. Yao, Generalized quasivariational inequality problems with discontinuous mappings, Math. Oper. Res., 20 (1995), 465-478.

36. J. Yu, Essential weak efficient solution in multiobjective optimization problems, J. Math. Anal. Appl., 166 (1992), 230-235.

37. Q. Yu and X. H. Gong, Existence of solutions for set-valued generalized strong vector equilibrium problems, J. Nanchang. Univ., 2 (2008), 103-107.

\author{
Bin Chen \\ Department of Mathematics \\ Sichuan University \\ Chengdu, Sichuan 610064 \\ P. R. China \\ E-mail: chenbin1984910@163.com
}


Nan-jing Huang

Department of Mathematics

Sichuan University

Chengdu, Sichuan 610064

P. R. China

E-mail: nanjinghuang@hotmail.com

Ching-Feng Wen

Center for General Education

Kaohsiung Medical University

Kaohsiung 807, Taiwan

E-mail: cfwen@kmu.edu.tw 